\title{
Adaptive Face Recognition System from Myanmar NRC Card
}

\author{
Ei Phyo Wai \\ University of Computer \\ Studies, Yangon, Myanmar
}

\author{
Myint Myint Sein \\ University of Computer \\ Studies, Yangon, Myanmar
}

\begin{abstract}
Biometrics is used for human recognition which consists of identification and verification. Identification applications are common when the goal is to identify criminals, terrorists, or other particularly through surveillance. Also, faces are integral to human interaction. Manual facial recognition is already used in everyday authentication applications. This paper focused on identification of personal information from National Registration Card and providing the information of NRC holder. Therefore there is no such face recognition system from low quality image of NRC card. Experimental results show a high recognition rate equal to $99.8 \%$ which demonstrated an improvement in comparison with previous methods using PCA.
\end{abstract}

\section{Keywords}

Face Recognition, Eigenfaces, Eigenvalues

\section{INTRODUCTION}

Pattern recognition plays a crucial role in real world applications. In an identification application, the biometric device reads a sample and compares that sample against every record or template in the database. Motivation of this paper is to identify authorized person for security system by using NRC card. To build high performance surveillance or information security systems, face recognition has been known as the key application attraction.

Face recognition has become an important issue in many applications such as security systems, credit card verification and criminal identification [2]. Much of the work in face recognition by computers has focused on detecting individual features such as the eyes, nose, mouth and head outline, and defining a face model by the position, size, and relationships among these features.

Eigenface approach is one of the simplest and most efficient methods in developing a system for Face Recognition. In eigenface approach, DiaPCA is used to reduce dimension and then the distance between two images is measured by using Euclidian distance for adaptive face recognition. If the distance is less than some threshold value, then it is considered as a known face else it is recognized as an unknown face [5].The approach transforms face images into a small set of characteristic feature images, called "eigenfaces", which are the principal components of initial training set of face images. Recognition is performed by projecting a new image into the subspace spanned by the eigenfaces and then classifying face by comparing its position in face space with the position of known individuals [4]. The difference between existing face recognition system and proposed face recognition system is described as follows. The proposed system can get the capable of extracting photo, character segmenting, character recognition have been done automatically from the scanned image based on the training positions. This system is considered the recognition of current image and source face image from NRC which is taken within 10 years. Therefore this proposed system can recognize human face by using low quality image from NRC card.

This system is different from other existing face recognition system and the first system of face recognition from NRC card. That provides the information of the Myanmar National Registration Card (NRC) holder. Moreover, check and the detail personal information is extracted from database.

The remainder of this paper is organized as follows: section 2 explains overview of the system and preprocessing module including the face detection stage, gray converting and histogram equalization. In section 3 we briefly review DiaPCA methods for dimension reduction, face recognition, eigenfaces and also the nearest neighbor classifier method for classification will be explained. Section 4 illustrates the experimental results and conclusion is explained in section 5 .

\section{OVERVIEW OF THE SYSTEM}

Proposed method consists of four parts: (i) image preprocessing that includes face region extraction, gray level converting, histogram equalization, (ii) dimension reduction using DiaPCA (iii) feature extraction using KNN classifier and (iv) compute distance between the current input image and training images. And then the information of desire person is displayed to determine the authorized person or unauthorized person. Therefore this face recognition system is very essential for security. The first step of preprocessing is face region extraction. And then enhancing state occurs. Face region extraction means the input face image is cropped from input image by using cropping tool. The current input image can be obtained from online (Webcam) or offline (Scanner). 


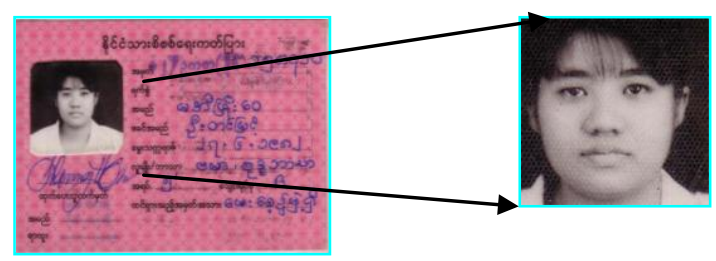

Cropped image

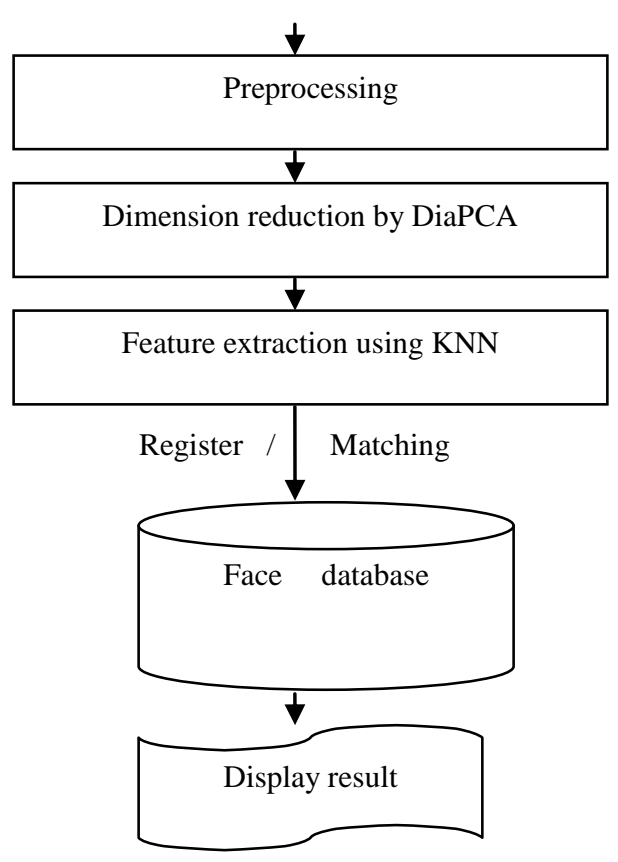

Fig 1: Overview of the proposed system

Enhancing states include the noise filtering, gray scale converting, and histogram equalization. After the face region is extracted from the scene, the input color image is converted to gray image and stored in database for processing.

After gray level converting, histogram equalization is occurred. Histogram equalization maps the input image's intensity values so that the histogram of the resulting image will have an approximately uniform distribution [7-10]. Through this adjustment, the intensity can be better distributed on the histogram.

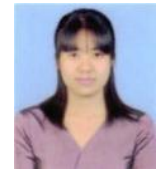

(a)

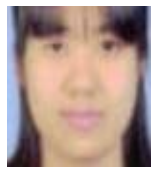

(b)

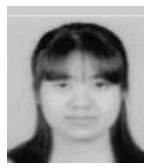

(c)

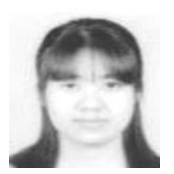

(d)

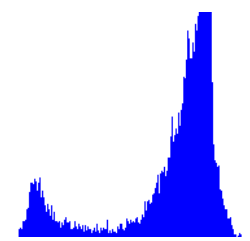

(e)

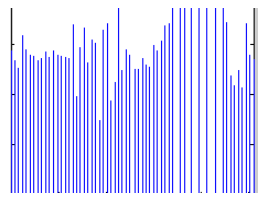

(f)
Fig 2: (a) input image(b) face region extraction (c) gray scale converting (d) histogram equalization (e) histogram for original image (f) histogram for equalized image

By histogram equalization, the local contrast of the object in the image is increased, especially when the usable data of the image is represented by close contrast values. Through this adjustment, the intensity can be better distributed on the histogram. This allows for areas of lower local contrast to gain a higher contrast without affecting the global contrast.

\section{PCA AND DIAPCA}

The Principal Component Analysis (PCA) is one of the most successful techniques that have been used in image recognition and compression. PCA is a statistical method under the broad title of factor analysis. The purpose of PCA is to reduce the large dimensionality of the data space to the smaller intrinsic dimensionality of feature space, which are needed to describe the data economically. This is the case when there is a strong correlation between observed variables. PCA can do prediction, redundancy removal, feature extraction, data compression, etc. Because PCA is a classical technique which can do something in the linear domain, applications having linear models are suitable, such as signal and image processing.

We have implemented PCA procedure in a training set of $M$ face images. Let a face image be represented as a two dimensional $N$ by $N$ array of intensity values, or a vector of dimension $N 2$. Then PCA tends to find a $M$-dimensional subspace whose basis vectors correspond to the maximum variance direction in the original image space. This new subspace is normally lower dimensional $(M \ll M \ll N 2)$. New basis vectors define a subspace of face images called face space. All images of known faces are projected onto the face space to find sets of weights that describe the contribution of each vector. To identify an unknown image, the image is projected onto the face space as well to obtain its set of weights. By comparing a set of weights for the unknown face to sets of weights of known faces, the face can be identified. PCA basis vectors are defined as eigenvectors of the scatter matrix $\mathrm{S}$ defined as:

$$
S=\sum_{i=1}^{M}(x i-\mu) \cdot(x i-\mu)^{\prime}
$$

where $\mu$ is the mean of all images in the training set and $x i$ is the $i^{\text {th }}$ face image represented as a vector $i$. As this face space is 
generated using eigenvectors of scatter matrix, sometimes this is also called as eigenspace.

The eigenvectors corresponding to nonzero eigenvalues of the covariance matrix produce an orthonormal basis for the subspace within which most image data can be represented with a small amount of error. The eigenvectors are sorted from high to low according to their corresponding eigenvalues. The eigenvector associated with the largest eigenvalue is one that reflects the greatest variance in the image. That is, the smallest eigenvalue is associated with the eigenvector that finds the least variance.

The vectors are also images, so called, eigenimages, or eigenfaces .They can be viewed as images and indeed look like faces. So describes the contribution of each eigenface in representing the facial image by treating the eigenfaces as a basis set for facial images. Face space forms a cluster in image space and PCA gives suitable representation.

Pattern recognition in high-dimensional spaces have pattern problems because of curse of dimensionality. Significant improvements can be achieved by first mapping the data into a lower-dimensional sub-space. The goal of PCA is to reduce the dimensionality of the data while retaining as much as possible of the variation present in the original dataset.

Our motivation for developing the DiaPCA method originates from an essential observation on the PCA. PCA only reflects the information between rows, which implies some structure information (e.g. regions of a face like eyes, nose, etc.) cannot be uncovered by it. We attempt to solve that problem by transforming the original face images into corresponding diagonal face images. Because the rows (columns) in the transformed diagonal face images simultaneously integrate the information of rows and columns in original images, it can reflect both information between rows and columns. DiaPCA may find some useful block or structure information for recognition in original images. DiaPCA directly seeks the optimal projective vectors from diagonal face images without image-to-vector transformation.

In the core of our system lies the Diagonal Principal Component Analysis (DiPCA) Algorithm [2], which is a tested and widely adopted for face recognition. DiaPCA can be subdivided into two components - PCA subspace training and PCA projection. During PCA subspace training, the rows of the pixels of an $\mathrm{N} 1 \times \mathrm{N} 2$ image are concatenated into a one dimensional 'image vector'. DiaPCA is a standard technique used to approximate the original data with lower dimensional feature vectors. The basic approach is to compute the eigen vectors of covariance matrix and approximate the original data by a linear combination of the leading eigenvectors.

During DiaPCA projection, a new face image vector is multiplied by the transformation matrix and projected to a point in a high dimensional DiaPCA subspace. In this case, the correlations among the projected images are minimized in order to facilitate easier classification [3]. The projected image is then saved as the face template of the corresponding user for future matching.

The eigenface-based face analysis that is based on the PCA for efficient face recognition [5]. In the training phase of the eigenface method, eigenvectors are calculated with a number of training faces. The computed eigenvectors are called as eigenfaces. Then, faces are enrolled in the face recognition system by their projection onto the eigenface space. In the recognition phase, an unknown input face can be identified by measuring the distances of the projected coefficients between the input face and the enrolled faces in database. The eigenface can be calculated by using the following equation.

$$
\mathrm{A}=\mathrm{u}^{1}{ }_{1}-\mathrm{v}, \ldots, \mathrm{u}^{1}{ }_{\mathrm{n}}-\mathrm{v}, \mathrm{u}^{\mathrm{P}}{ }_{1}-\mathrm{v}, \ldots, \mathrm{u}^{\mathrm{P}}{ }_{\mathrm{n}}-\mathrm{v}
$$

To calculate eigenfaces, first calculate average face (v) . And then collect differences between training images and average face in matrix $\mathrm{A}$ ( $\mathrm{M}$ by $\mathrm{N}$ ) where $\mathrm{M}$ is the number of pixels and $\mathrm{N}$ is the number of images .

$$
\mathrm{C}=\mathrm{AA}^{\mathrm{T}}
$$

The eigenvectors of covariance matrix $\mathrm{C}$ ( $\mathrm{M}$ by $\mathrm{M}$ ) give the eigenfaces.

\subsection{Nearest Neighbor Classification}

Classification (similarity search) is a very crucial step in any pattern recognition application. One of the most popular nonparametric techniques is the Nearest Neighbor classification (NNC). KNN classifier has many advantages. This is easy to compute and very efficient. $\mathrm{KNN}$ is very compatible and obtain less memory storage. So it has good discriminative power. KNN is very robust to image distortions (e.g. rotation, illumination). Therefore KNN can provide the components that describe the highest variance and produce good result. So this research can produce good result by combining DiaPCA and KNN ( $\mathrm{K}^{\text {th }}$ nearest neighbor classifier).

\subsection{Face Recognition}

Dimension of an image space is so high that it is often impractical to deal with all the data of images in their own dimensions. DiaPCA enables to optimally reduce the dimensionality by constructing the eigenface space that is composed of the eigenvectors [5]. A face image is transformed by projecting it onto the eigenface space. And then the input face image is compared the eigenface of database for intelligent face recognition.

Face recognition has many applicable areas. The most useful applications contain crowd surveillance, video content indexing, personal identification (eg. driver's license), entrance security, etc. The main idea of using DiaPCA for face recognition is to 
reduce the large dimension constructed from 2-D facial image into the compact principal components of the feature space. This can be called eigenspace projection. Once the eigenfaces have been computed, several types of decision can be made depending on the application.

The images with a low correlation can be rejected or these two issues are altogether addressed by categorizing following four regions:

- Near face space and near stored face $=>$ known faces

- Near face space but not near a known face => unknown faces

- $\quad$ Distant from face space and near a face class => nonfaces

- $\quad$ Distant from face space and not near a known class $=>$ non-faces

Since a face is well represented by the face space, its reconstruction should be similar to the original and the reconstruction error will be small. Non-face images will have a large reconstruction error which is larger than some threshold. There have been many methods proposed for face recognition. And one of the key components of any methods is a facial feature extraction. Facial feature could be a gray-scale-image.

\section{EXPERIMENTAL RESULTS}

In this part, the system uses thousands of face images as training database. Experimental results using MATLAB are demonstrated in this paper to verify the viability of the proposed face recognition method. At first the system manually crops the face image from the input images in order to remove the undesired information and have only face image details. The input image may be the current color image or the scanned image from the NRC card.
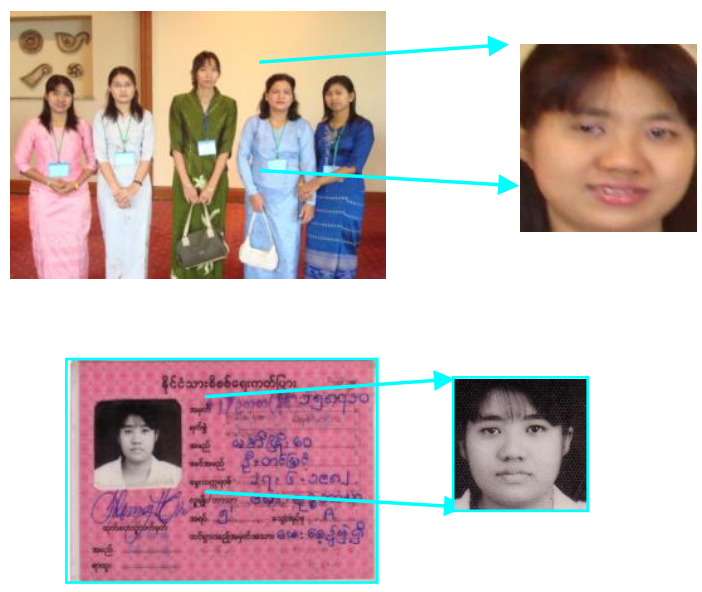

Fig 3: Region of Interest
Number of eigen-vector depend on the application and accuracy, it is clear that if the system compute large number of eigenvectors accuracy of the method improved but computational complexity increased in this step and next step. In this stage, the system computed 100 most significant eigenvectors and related eigen-faces.

This system is implemented by MATLAB. The graphic user interface is applied for developed system implementation. The main menu of the developed program is described in figure 4.

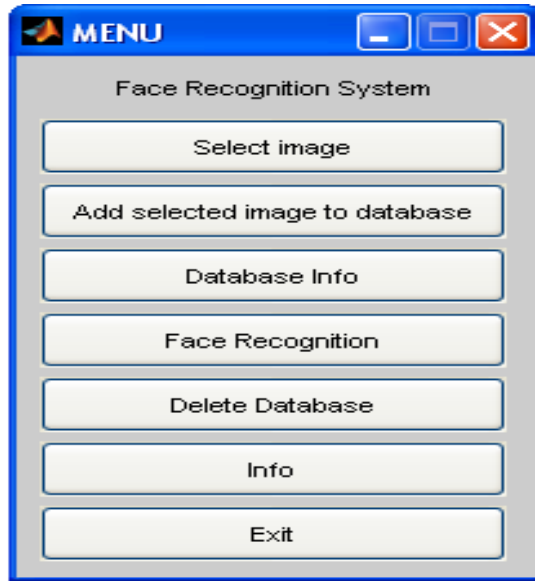

Fig 4. The main menu of the program

The image selection box can be seen by clicking 'select image button'. And then the desire image can be selected from the image selection box. And the image can be added to database or deleted from database. The user can see the information of database and can recognize the desire person.

Additional image is stored in database with the id number by clicking 'Add selected image to database' button from the MENU box. The information of database is shown by selecting 'Database Info' button and show the number of images stored in database.

The main process of this paper is face recognition by clicking 'Face Recognition' button. First the desire image can be selected from the image selection box and click 'Face Recognition'. And then the output can be shown illustrated in figure 5 . 


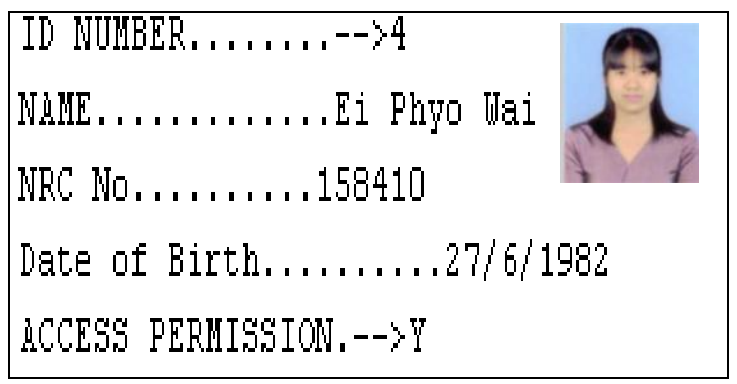

Fig 5. The result of the program

The next processes are delete database, information, and exit the program. When click delete database button, delete the entire database and can see the number of stored images by clicking information button. When click Exit button, can exit MATLAB platform.

\begin{tabular}{|c|c|c|c|}
\hline No. & Extraction & Rate & $(\%)$ \\
& Method & $(\%)$ & \\
\hline 1. & PCA only & 93.5 & 2.9 \\
\hline 2. & PCA+SVM & 96.5 & 1.32 \\
\hline 3. & PCA+LDA & 98.7 & 1.92 \\
\hline 4. & DiaPCA+KNN & 99.8 & 1.2 \\
\hline
\end{tabular}

Fig 6. Comparison of different approaches

According to this comparison, the proposed method has the best recognition rate and the least error rate based on thousands of test face images.

\section{CONCLUSION}

In this paper, a new Face recognition method is presented. The new method was considered as a combination of DiaPCA and KNN classifier. This system can be used to construct efficient face recognition method with a high recognition rate. This research chose 10 persons and considered 40 training image and 20 test image for each person (totally 400 training and 200 test face images). Experimental results show a high recognition rate equal to $99.8 \%$ (in average one misclassification for each 200 face images) which demonstrated an improvement in comparison with previous methods using PCA. The new face recognition algorithm can be used in many applications such as security methods.

\section{REFERENCES}

[1] A. Jain, R. Bolle, S. Pankanti Eds, "BIOMETRIC - Personal Identification in Networked Society", Kluwer AcademicPublishers, Boston/ Dordrecht/ London, 2007.

[2] B. Moghaddam, "Principal manifolds and probabilistic subspaces for facel recognition", IEEE Trans. pattern Anal. Machine Intel., Vol. 24, No. 6, PP. 780-788, 2008.

[3] D.L. Swets and J.J. Weng, "Using Discriminant Eigen features for image retrieval", IEEE Trans. Pattern Anal. Machine Intel, vol. 18, PP. 831-836, Aug. 2009.

[4] H. Othman, T. Aboulnasr, " A separable low complexity 2DHMM with application to face recognition" IEEE Trans. Pattern. Anal. Machie Inell., Vol. 25, No. 10, PP. 1229-1238, 2008.

[5] J. Creed, A Abbott, Optimization of Color Conversion for Face Recognition, EURASIP Journal on Applied Signal Processing, 4: 522-529, 2010.

[6] J. R. Solar, P. Navarreto, " Eigen space-based face recognition: a comparative study of different approaches, IEEE Tran. , Systems man And Cybernetics- part c: Applications, Vol. 35, No. 3, 2005.

[7] M. Turk, A. Pentland, "Eigen faces for face recognition", Journal cognitive neuroscience, Vol. 3, No.1, 2008.

[8] O.Deniz, M. Castrill_on, M. Hern_andez, "Face recognition using independent component analysis and support vector machines", Pattern Recognition letters, Vol. 24, PP. 21532157, 2010.

[9] P.N. Belhumeur, J.P. Hespanha, and D. J. Kriegman, "Eigenfaces vs. Fisher faces: Recognition using class specific linear projection", IEEE Trans. Pattern Anal. Machine Intel., vol. 19, PP. 711-720, may 2008.

[10] S. H Ou, Q. L. Wang, Z. Y. Zhu. The Application and Technology of Digital Image Processing. Beijing: Tsinghua Press, 2010. 